\title{
Bromodomain inhibition halts heart failure
}

Heart failure (HF) is the leading cause of mortality in modern society. It is a consequence of pathological remodelling of the heart, which involves cardiac hypertrophy (a robust predictor of subsequent $\mathrm{HF}$ and death), fibrosis and inflammation. At the molecular level, HF has been associated with chromatin hyperacetylation. Now, reporting in Cell, Anand et al. show that the BET family bromodomain proteins (BETs), a family of acetyl-lysine reader proteins, are central to $\mathrm{HF}$ pathogenesis and that they are promising targets for the prevention of HF.

The remodelling that precedes HF is induced by haemodynamic and neuronal stressors. These activate a signal transduction cascade that converges on a defined set of

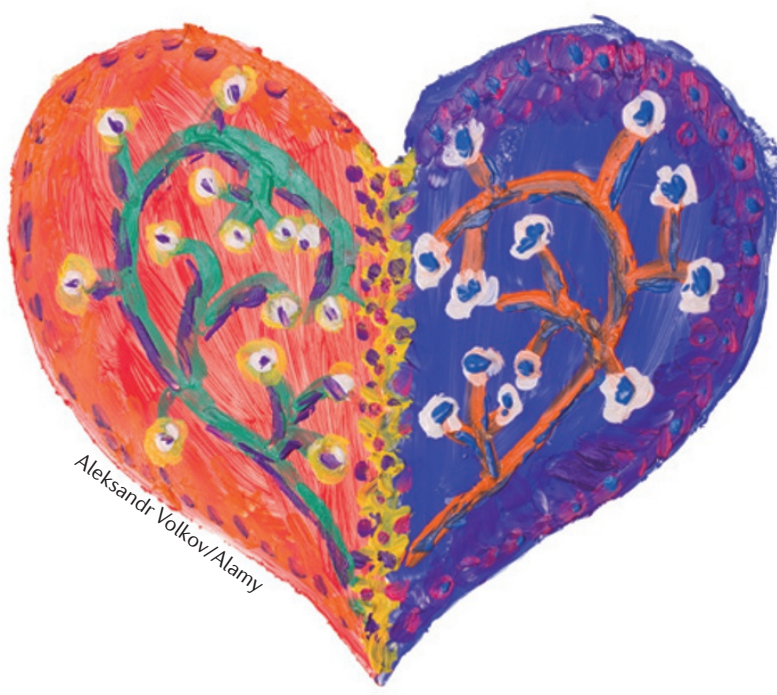

transcription factors, and pathological changes in cardiomyocytes occur through an interplay between these transcription factors and epigenetic changes in chromatin structure. Both epigenetic 'writer' proteins (histone acetyltransferases) and epigenetic 'erasers' (histone deacetylases) have been implicated in cardiac development and disease. However, the role of 'readers' (bromodomain proteins) was less well defined.

The group utilized the recently developed first-in-class inhibitor JQ1, which displaces BETs from chromatin, resulting in the suppression of downstream signalling to RNA polymerase II (Pol II). Investigating the role of BETs in an in vitro model of neurohormonally induced heart disease, the authors showed that nanomolar doses of JQ1 significantly blocked phenylephrine-mediated hypertrophy of cardiomyocytes and pathological gene induction. Similar results were achieved through knockdown of the BET bromodomain-containing protein 4 (BRD4), which is highly expressed in cardiac tissues. Gene expression profiling of cultured cardiomyocytes in the presence or absence of JQ1 showed that the drug abrogated the induction of a substantial subset of phenylephrine-induced genes.

In vivo experiments of $\mathrm{HF}$ induced in mice via phenylephrine or haemodynamic stress (using the surgical method of transverse aortic constriction (TAC)) showed that JQ1 protected against several pathological changes, including cardiac hypertrophy. Importantly, no toxicity or effects on systemic blood pressure were observed.

Detailed transcriptional analysis of cardiac tissue from mice that were either TAC- or sham-operated and treated with either JQ1 or vehicle revealed that BET inhibition suppresses a pathological cardiac gene expression programme. Importantly, the targets of BETs in the mouse TAC model were also found to be relevant in human HF.

Further molecular analysis revealed that BETs play a crucial role in chromatin-mediated signal transduction to Pol II, co-activating transcription factor networks that are known to be causal in HF pathogenesis, including nuclear factor of activated T cells (NFAT), nuclear factor $-\kappa \mathrm{B}(\mathrm{NF}-\kappa \mathrm{B})$ and the transcription factor GATA4. Moreover, BETs were found to function, in part, by promoting transcriptional pause release (and thereby re-activating transcription) during pathological stress.

This study implicates epigenetic readers in cardiac biology and suggests that the manipulation of cardiac gene control via the targeting of chromatin-dependent signal transduction could potentially abrogate pathological gene expression and $\mathrm{HF}$ progression.

Alexandra Flemming

ORIGINAL RESEARCH PAPER Anand, P. et al. BET bromodomains mediate transcriptional pause release in heart failure. Cell 154, 569-582 (2013) 